\title{
Real-time Amyloid Aggregation Monitoring with a Photonic Crystal-based Approach
}

\author{
Sara Santi, ${ }^{[a, b]}$ Valeria Musi, ${ }^{[a]}$ Emiliano Descrovi, ${ }^{[c]}$ Vincent Paeder, ${ }^{[a]}$ Joab Di Francesco, ${ }^{[a]}$ \\ Lubos Hvozdara, ${ }^{[\mathrm{ac}]}$ Peter van der Wal, ${ }^{[\mathrm{d}]}$ Hilal A. Lashuel, ${ }^{[\mathrm{e}]}$ Annalisa Pastore, ${ }^{[\mathrm{f}]}$ \\ Reinhard Neier, ${ }^{*[b]}$ and Hans Peter Herzig ${ }^{*[a]}$
}

\begin{abstract}
We propose the application of a new label-free optical technique based on photonic nanostructures to real-time monitor the amyloid-beta 1-42 (A $\beta(1-42))$ fibrillization, including the early stages of the aggregation process, which are related to the onset of the Alzheimer's Disease (AD). The aggregation of $A \beta$ peptides into amyloid fibrils has commonly been associated with neuronal death, which culminates in the clinical features of the incurable degenerative AD. Recent studies revealed that cell toxicity is determined by the formation of soluble oligomeric forms of $A \beta$ peptides in the early stages of aggregation. At this phase, classical amyloid detection techniques lack in
\end{abstract}

sensitivity. Upon a chemical passivation of the sensing surface by means of polyethylene glycol, the proposed approach allows an accurate, real-time monitoring of the refractive index variation of the solution, wherein $A \beta(1-42)$ peptides are aggregating. This measurement is directly related to the aggregation state of the peptide throughout oligomerization and subsequent fibrillization. Our findings open new perspectives in the understanding of the dynamics of amyloid formation, and validate this approach as a new and powerful method to screen aggregation at early stages.

\section{Introduction}

The misfolding and aggregation of specific proteins have been recognized as the cause of widespread degenerative patholo-

[a] S. Santi, Dr. V. Musi, Dr. V. Paeder, J. Di Francesco, Dr. L. Hvozdara, Prof. H. P. Herzig

Optics \& Photonics Technology Laboratory

Institute of Microengineering

École Polytechnique Fédérale de Lausanne (EPFL)

$\mathrm{CH}-2000$ Neuchâtel (Switzerland)

E-mail: hanspeter.herzig@epfl.ch

[b] S. Santi, Prof. R. Neier

Laboratory of Organic Chemistry

Institute of Chemistry

University of Neuchâtel

CH-2000, Neuchâtel (Switzerland)

E-mail: reinhard.neier@unine.ch

[c] Dr. E. Descrovi

Dipartimento di Scienza Applicata e Tecnologia

Politecnico di Torino

10129, Torino (Italy)

[d] Dr. P. van der Wal

Sensors, Actuators and Microsystems Laboratory

Institute of Microengineering

École Polytechnique Fédérale de Lausanne (EPFL)

$\mathrm{CH}-2000$ Neuchâtel (Switzerland)

[e] Prof. H. A. Lashuel

Laboratory of Molecular and Chemical Biology of Neurodegeneration Brain Mind Institute

École Polytechnique Fédérale de Lausanne (EPFL)

$\mathrm{CH}-1015$ Lausanne (Switzerland)

[f] Prof. A. Pastore

MRC, National Institute for Medical Research

The Ridgeway, London

NW7 1AA (UK)

Supporting information for this article is available on the WWW under http://dx.doi.org/10.1002/cphc.201300633. gies such as Alzheimer's and Parkinson's diseases. ${ }^{[1]}$ In the specific case of Alzheimer's disease (AD), the neuronal and synaptic loss has been commonly identified with the presence of senile plaques of extracellular highly fibrillogenic amyloid-beta peptides $(A \beta)$. $A \beta$ peptides, the longest one being $A \beta(1-42)$, are produced as soluble monomers. The prevailing hypothesis is that cell toxicity, associated with neuronal death, is triggered by intermediate soluble oligomeric forms of $A \beta$ peptide developing at a very early stage of aggregation, rather than by the final insoluble plaques composed of $A \beta$ fibrils. ${ }^{[2]}$

$A \beta$ aggregation is thought to proceed through the nucleation-polymerization model. ${ }^{[3]}$ This model consists of an initial rate-limiting nuclei formation, described as "lag phase", corresponding to the formation of toxic oligomeric species at the early stages of $A \beta$ aggregation, followed by an elongation/ polymerization phase which leads to the formation of fibrils. One of the major challenges for $A D$ research is to detect the early events of aggregation since the classical detection methods, such as Congo Red and thioflavin T staining, transmission electron microscopy (TEM), X-ray diffraction and classical NMR are all too insensitive to detect the minute soluble species formed during the lag phase. ${ }^{[4]}$ Prompt and sensitive detection would also be crucial to allow $A D$ early diagnosis and design of powerful therapeutic treatments.

In this work, we exploit the unique properties of an optical label-free refractometric sensing platform to propose a novel approach for investigating the initial lag-phase of $A \beta(1-42)$ aggregation. Our sensing approach allows the real-time optical detection of local refraction index changes occurring as aggregation takes place. The method is based on the optical interrogation of a dielectric multilayer (one-dimensional photonic 

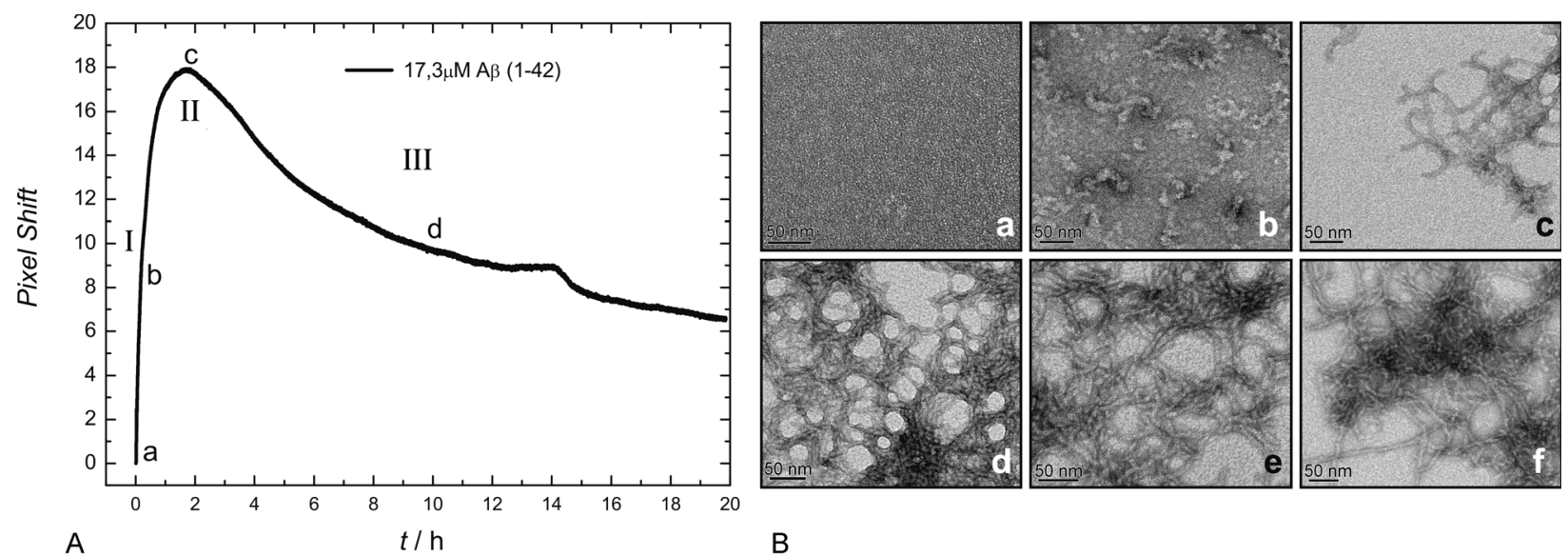

Figure 1. A) Refractive index variation $\Delta n$, in pixel shift, of an $\mathrm{A} \beta(1-42)$ monomer peptide solution incubated at $37^{\circ} \mathrm{C}$ at the initial concentration of $17,3 \mu \mathrm{M}$ in $10 \mathrm{~mm}$ Tris $\mathrm{HCl}, \mathrm{pH}$ 7.4. Roman numbers refer to the three distinct zones of the $\Delta n$ monitoring, as described in the text. The lower-case letters refer to the TEM micrographs collected at different stages of aggregation, during the $\Delta n$ monitoring. B) TEM micrographs of $A \beta(1-42)$ monomer at the initial concentration of $17 \mu \mathrm{M}$ (in $10 \mathrm{~mm}$ Tris $\mathrm{HCl}, \mathrm{pH} 7.4$ ) at: a) $t=0, T=4{ }^{\circ} \mathrm{C}$; b) $t=30 \mathrm{~min}, T=37^{\circ} \mathrm{C}$; c) $t=2 \mathrm{~h}, T=37^{\circ} \mathrm{C}$; d) $t=10 \mathrm{~h}, T=37^{\circ} \mathrm{C}$; e) $t=24 \mathrm{~h}, T=37^{\circ} \mathrm{C}$; f) $t=48 \mathrm{~h}$, $T=37^{\circ} \mathrm{C}$. The morphological changes in the peptide solutions during incubation are displayed.

crystal) sustaining an electromagnetic surface wave. ${ }^{[5]}$ The sensing technique is reminiscent of surface plasmon resonance (SPR). Instead of having a surface plasmon propagating on a thin metallic film, an evanescent wave called "Bloch surface wave" (BSW) is coupled on a purely dielectric multilayer structure ${ }^{[6]}$ Depending on the materials used and the layout of the periodic dielectric multilayer, the BSW can be produced in a wide spectral range, from the visible to the near-infrared. ${ }^{[7]}$ This spectral tunability represents one of the main advantages of BSW-based optical transduction systems, as compared to SPR. In addition, BSWs present very narrow resonances that can increase the resolution and improve the limit of detection in label-free detection schemes. BSWs generated on dielectric multilayers are sensitive to external perturbations of the refractive index close to the surface of the photonic multilayer. ${ }^{[8]}$

In the following, we report on a proof of principle of the BSW-based detection technique for sensing protein aggregation by monitoring in real time the refractive index variation $(\Delta n)$ of an aqueous solution containing the $A \beta(1-42)$ peptide during early aggregation and fibril formation. The multilayer surface is directly contacted with the probed aqueous medium and the sensing chamber is positioned vertically (see Experimental section). Hence, the measurements using BSWs monitor a local variation of the refractive index of the solution, which is directly related to the depletion of the concentration of the A $\beta(1-42)$ monomeric form during aggregation.

\section{Results and Discussion}

\section{1. $A \beta(1-42)$ Aggregation Sensing with Bare Multilayer Sur- face}

The monomeric $A \beta(1-42)$ peptide was incubated at $37^{\circ} \mathrm{C}$ in the fluidic chamber in the presence of an initial bare (non-functionalized) multilayer. (For details on sample preparation and experimental setup see the Experimental Section.) The mea- surement consisted in time-monitoring the angular position of a BSW resonance dip upon laser illumination through a prism. $A$ representative aggregation curve obtained during incubation of the $A \beta(1-42)$ peptide is shown in Figure $1 A$. The variation in the refractive index $\Delta n$ of the initially monomeric $A \beta(1-$ 42) solution is monitored as a function of time by taking the BSW resonance position on a linear CMOS camera. We observed a sudden increase of the signal followed by a slow exponential decay of $\Delta n$ of the solution within the time frame where other techniques show a lag time. ${ }^{[3 c]}$ The $\Delta n$ decrease is in accordance with the $A \beta(1-42)$ monomer depletion due to aggregation. The BSW-assisted monitoring was compared with TEM imaging data to allow the detection of the $A \beta(1-42)$ species and their corresponding morphologies before and during aggregation. ${ }^{[9]}$ TEM imaging was performed on the sample probed at different stages of aggregation, thus revealing the morphological evolution of the peptide from a monomeric (Figure 1B, micrograph a), to an oligomeric and pre-fibrillar stage (Figure 1B, micrographs b-d), and to a non-soluble aggregate fibrillar conformation (Figure $1 \mathrm{~B}$, micrographs e and $\mathrm{f}$ ).

Many different models of the aggregation process have been proposed, but to the best of our knowledge there is no direct experimental evidence that allows assessment of the events that we report shortly after the monomer injection. A simple empirical model describing the variation of $\Delta n$ as sensed by the BSW involves the contribution from two terms, shown in Equation (1):

$\Delta n=\Delta n_{\mathrm{s}}+\Delta n_{\mathrm{b}}$

wherein $\Delta n_{\mathrm{s}}$ is the refractive index variation due to surface adsorption/desorption processes, and $\Delta n_{\mathrm{b}}$ is the refractive index variation occurring in the bulk solution, as observed within the evanescent tail of BSWs. Since our multilayer presents a bare silicon oxinitride surface, $\Delta n_{s}$ is mainly due to the hydrophobic adsorption of the $A \beta(1-42)$ peptide onto the surface. Instead, 
$\Delta n_{\mathrm{b}}$ refers mainly to the variation of the $\mathrm{A} \beta(1-42)$ monomer concentration in the bulk solution. The decrease of the monomer concentration reveals the formation of non-soluble aggregates. The final result of the $A \beta(1-42)$ aggregation process, the precipitate of the non-soluble fibers, statistically accumulates at the bottom of the vertically positioned sensing chamber, away from the BSW probe.

The overall time evolution of the refractive index value $\Delta n$, as shown in Figure $1 \mathrm{~A}$, can be divided into three distinct stages (labeled with Roman numbers in Figure $1 \mathrm{~A}$ ) that are discussed in the following:

I) An initial period characterized by a steep increase of $\Delta n$ during the first approximately $2 \mathrm{~h}$ of the experiment. This is consistent with the adsorption or physisorption of the $A \beta(1-42)$ peptide in an adhesion-competent conformation, onto the silicon oxinitride surface. This event produces a local increase of $\Delta n_{s}$. This surface-loading mechanism hampers the direct observation of the $\Delta n_{\mathrm{b}}$ decrease due to peptide aggregation in this very early stage. Nevertheless, at this specific early stage, the changes of the concentrations in the bulk solution are expected to be negligible.

II) A maximum amplitude of the curve, attained at $\sim 2 \mathrm{~h}$ and characterized by the amount of the overall $\Delta n$ change as measured from the start of the experiment. The reaching of the maximum refractive index value can be associated to an equilibrium condition due, for example, to the saturation of the surface and a decreased availability of free adhesion-competent peptides because of a simultaneous polymerization occurring in the solution.

III) A slow and steady decrease of $\Delta n$ as observed after passing the maximum. The exponential decrease of the refractive index can be possibly ascribed to two processes: the detachment of the peptide from the surface, and/or the formation of fibrillar structures according to the aggregation mechanism, and thereby depleting the bulk solution. Both of them lead to a decrease of $\Delta n$. In the following we will show that different conformational species of $A \beta(1-42)$ exhibit different affinities for the surface.

\subsection{A $\boldsymbol{\beta}(1-42)$ Aggregation Sensing with PEG-ylated Multi- layer Surface}

For a better understanding of the type of process observed during phase III in Figure $1 \mathrm{~A}$, we explored an alternative approach based on a chemical coating of the multilayer. It has been shown ${ }^{[10]}$ that polyethylene glycol (PEG) of a molecular weight of $750 \mathrm{Da}$, when attached to a glass-like surface, forms a brush-like self-assembled monolayer (SAM). The SAM thickness is reported to be $50-70 \AA$. The chemical modification of the multilayer surface by means of PEG was performed to obtain an almost inert surface. ${ }^{[11]}$ In this case, the modified multilayer surface should therefore sense the process of $A \beta(1-$ 42) aggregation with a less significant influence of the peptide interactions with the surface.
The monitoring of $A \beta(1-42)$ monomer aggregation with a PEG-ylated multilayer is shown in Figure $2 \mathrm{~A}$, where it is referred to as $M_{P E G}$. The $M_{P E G}$ curve reveals an initial positive pixel
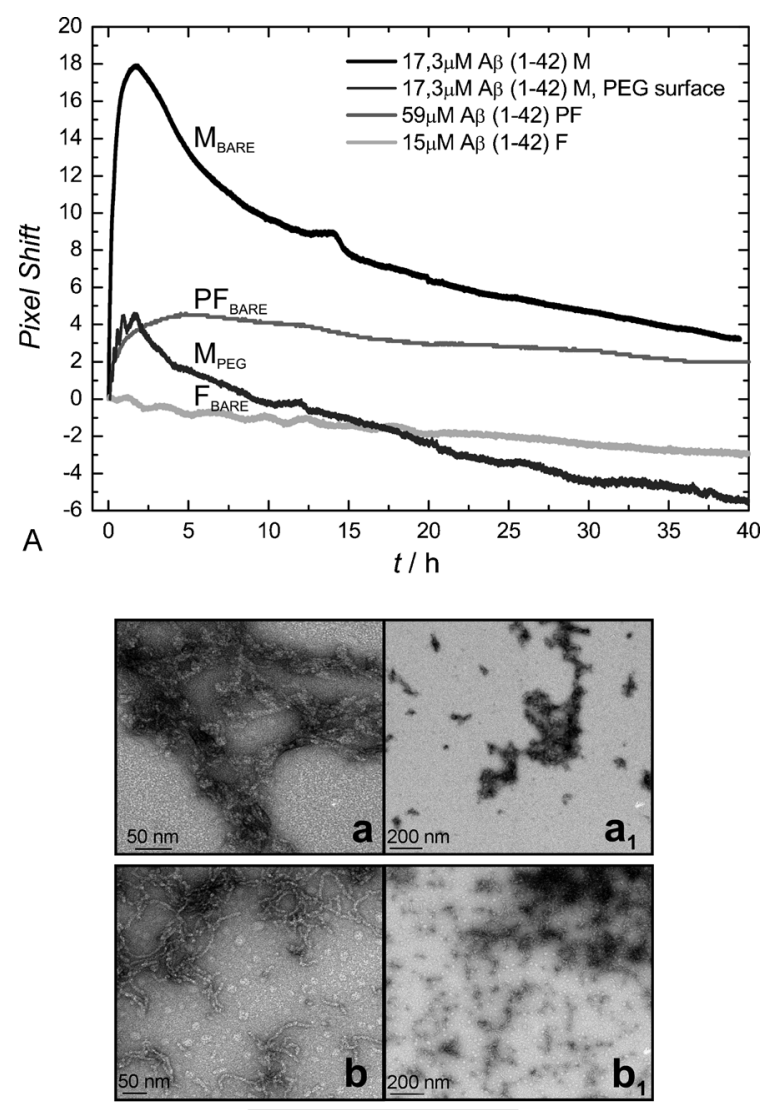

B

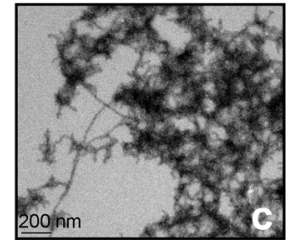

Figure 2. A) Real-time refractive index variation of $A \beta(1-42)$ peptides, incubated at $37^{\circ} \mathrm{C}$, at different initial aggregation states and experimental conditions: $M_{\text {BARE }}, 17.3 \mu \mathrm{M}$ initially monomeric peptide in contact with a bare multilayer, $\mathrm{M}_{\mathrm{PEG}}, 17.3 \mu \mathrm{M}$ initially monomeric peptide in contact with a PEGylated multilayer, $\mathrm{PF}_{\mathrm{BARE}}, 59 \mu \mathrm{M}$ protofibril in contact with a bare multilayer, and $F_{B A R E}$, fibril form of the peptide (produced incubating a $15 \mu \mathrm{M}$ monomeric sample at $37^{\circ} \mathrm{C}$ for $48 \mathrm{~h}$ ) in contact with a bare multilayer. B) TEM micrographs of: protofibril at the initial concentration of $59 \mu \mathrm{M}$ at: $\mathrm{a}$ and a1) $t=0 \mathrm{~h}, T=4{ }^{\circ} \mathrm{C}$; b and b1) $t=24 \mathrm{~h}, T=37^{\circ} \mathrm{C}$; fibril at: c) $t=0 \mathrm{~h}, T=4{ }^{\circ} \mathrm{C}$.

shift that is four times smaller as compared to the case of the same experiment with a bare multilayer, referred to as $M_{B A R E}$ in Figure $2 \mathrm{~A}$. In fact, on the PEG-ylated multilayer surface a smaller amount of adhesion-competent peptide is adsorbed, causing a smaller $\Delta n_{\mathrm{s}}$ increase. After $10 \mathrm{~h}$ of incubation, this curve reaches negative values for the BSW resonance shift. This means that the refractive index of the solution, as sensed by the BSW, at this stage has become lower compared to the value measured at the initial stage of the incubation. This does not happen when incubating the $A \beta(1-42)$ monomer with 
a bare surface because of the shielding due to the initial massloading mechanism. Therefore, the presence of a PEG coating effectively decreased the loading effect, thus making the BSW mainly sensitive to bulk fibrillization. After an incubation time between 8 and $12 \mathrm{~h}$, the $M_{P E G}$ and $M_{B A R E}$ curves show decreasing slopes that present a similar rate. Therefore, for long incubation time, (over 8 and $12 \mathrm{~h}$ ), the temporal evolution of the refractive index change is in general related to the bulk of the solution only, $\Delta n_{b}$, where the fibrillization of the $A \beta(1-42)$ peptide occurs, regardless of the surface functionalization. The surface-loading process only results in a decrease of sensitivity, still allowing estimating the time rate of $\Delta n_{\mathrm{b}}$.

\subsection{Protofibrillar and Fibrillar $A \beta(1-42)$ Aggregation}

We finally considered $A \beta(1-42)$ samples containing defined populations of $A \beta$ species, including protofibrillar and fibrillar forms of $A \beta(1-42)$, prepared as described in the Experimental Section. The purpose of these measurements was to try identifying the different aggregation states with the sensograms obtained along the amyloid pathway of $A \beta(1-42)$ samples. Protofibril samples are heterogeneous prefibrillar aggregates of different morphologies and sizes, ${ }^{[9]}$ reported to be generally unable to assemble into fibrils in the absence of the $A \beta(1-42)$ monomer, $^{[12]}$ and showing low induced fluorescence response upon binding ThT. The mature aggregates, the fibrils, filamentous aggregates presenting considerable $\beta$-sheet structure, strongly bind ThT (see the Supporting Information, Figure S1). The resulting profiles of protofibrils $\left(\mathrm{PF}_{\mathrm{BARE}}\right)$ and fibrils $\left(\mathrm{F}_{\mathrm{BARE}}\right)$, incubated at $37^{\circ} \mathrm{C}$ in the BSW sensing cell with a bare multilayer, are shown in Figure $2 \mathrm{~A}$, and compared to the profile of the monomeric peptide incubation $\left(\mathrm{M}_{\mathrm{BARE}}\right)$. Monomeric, protofibrillar and fibrillar forms of $A \beta(1-42)$ peptide present three distinct curves for the evolution of the refractive index over time. Regardless of the starting material used, the overall $\Delta n$ decreases on the long run. The variation of the refractive index of the incubated protofibrils and fibrils is smoother with respect to the case of monomeric species, during incubation at $37^{\circ} \mathrm{C}$. Previous studies $^{[9,12 b]}$ suggest that, $A \beta(1-42)$ fibrils and, specially, purified protofibril species present a less pronounced tendency to further assembly, being already embedded in an organized aggregation pattern. The TEM micrographs reveal a slow evolution of the $A \beta(1-42)$ protofibril during a 24-hour period of incubation, as shown in micrographs $a, a_{1}$ and $b, b_{1}$ in Figure $2 B$.

Concerning the surface loading effect, we do not report an appreciable $\Delta n$ increase when mature fibrils, identified by TEM imaging (Figure 2B, micrograph $\mathrm{C}$ ), are incubated in the BSW sensing cell. This evidence allows inferring that surface adsorption of mature fibrils can be safely neglected in this specific case. Since fibrils were obtained incubating a purified monomeric $A \beta(1-42)$ sample at $37^{\circ} \mathrm{C}$ for $48 \mathrm{~h}$, the incubation of these mature aggregates can be even considered as a continuation of the measurement of the $A \beta(1-42)$ monomer incubation, after $48 \mathrm{~h}$, unaffected by any significant peptide interaction with the multilayer. More interestingly, BSW monitoring of the protofibrillar $A \beta(1-42)$ form shows no substantial surface adsorption. This observation is indicated by a minute increase of the $\Delta n$ value during the first period of the incubation, as compared to the $\Delta n$ of the $M_{\text {BARE }}$ profile. The observed increase stays small especially if one takes into account that a four times smaller concentration of monomer was used in the experiment.

Accordingly, the surface adsorption mechanism onto the unfunctionalised multilayer is found to be a process associated with and depending on the $A \beta(1-42)$ peptide in a specific aggregation form. Protofibrils seem to be less prone to be adsorbed onto the surface. The species that are strongly adsorbed onto the multilayer oxinitride surface have to be in a precise pre-fibrillar conformation, with a lower molecular weight than the average weight attributed to protofibrils.

\section{Conclusions}

The present work describes how a photonic crystal-based optical platform can be applied to monitor the $A \beta(1-42)$ peptide aggregation during the early events of the fibrillization process that leads to Alzheimer's disease. By sensing the refractive index change of the peptide solution, this approach provides unprecedented information covering the transition period from a homogenous dispersion, the monomer solution, to a heterogeneous suspension containing fibrillar amyloids. The presented photonic crystal-based approach provides relevant information on the aggregation of $A \beta(1-42)$ peptides during the nucleation process, the silent lag phase, where other classical amyloid detection methods are limited by their sensitivity.

As reported in the literature, ${ }^{[13]}$ the $A \beta(1-42)$ peptide undergoes major morphological changes during the aggregation process. The variation of the $A \beta(1-42)$ secondary structure has been extensively demonstrated ${ }_{r}^{[14]}$ especially in the transition from the monomeric form to the soluble oligomeric forms and these changes vary the water-exposed surface of the peptide. ${ }^{[15]}$ We suggest that the $A \beta(1-42)$ peptide desorption from the sensing surface, confirmed by the PEG-coating passivation experiment, should be caused by a variation in the secondary structure of the species initially adsorbed onto the multilayer surface. In the pathway from the oligomeric forms to the mature fibrils the structures become more compact and expose a different pattern that has less affinity to the surface. The TEM micrographs in Figure 1B are in good agreement with this hypothesis, showing the formation of oligomeric protofibrils from lower to higher molecular weight, in correspondence with the establishment of the maximum amplitude of the $\Delta n$ signal for the monomeric profile $\mathrm{M}_{\mathrm{BARE}}$. The presented results support the hypothesis that the interaction with the unfunctionalized bare surface may be determined by a specific early molecular structure variation. An analogous event has been reported by Yagi et al., ${ }^{[16]}$ whose work suggests the formation of oligomeric $A \beta$ seeds attached to a quartz surface.

The results obtained by monitoring the $A \beta(1-42)$ aggregation in the presence of a passivated surface allowed demonstrating that the interaction with the silicon oxinitride multilayer surface is not fundamentally modifying the mechanism of the aggregation. Without PEG, for long incubation time, the mass-loading mechanism has definitely reached a stationary 
condition, resulting in the formation of an overlayer of $A \beta(1$ 42) peptide steadily adsorbed on the surface. The presence of such an overlayer produces a shielding effect leading to a general overestimation of the solution refractive index. With PEG, almost no overlayer is formed on the surface and the BSW resonance position can be more directly related to the refractive index of the solution.

Despite the technical limitations imposed by the prototype system, our approach reveals a high potential that might overcome some of the limitations in other techniques. ${ }^{[17]}$ The presented photonic crystal-based approach is a novel promising candidate for direct, label-free and real-time monitoring of the $A \beta$ transitions during oligomerization at early stages. In perspective, this innovative amyloid-sensing strategy can be exploited to screen for molecular factors that influence the oligomerization process. Furthermore, the photonic crystal-based sensing can serve as a direct screening tool for new potential inhibitors of $A \beta(1-42)$ toxic oligomers and early nucleation events.

\section{Experimental Section}

\section{A $\beta(1-42)$ Monomer, Protofibril and Fibril Preparation and Characterization}

The $A \beta(1-42)$ amyloid peptide for all the experiments was provided by the group of Hilal Lashuel, Laboratory of Molecular and Chemical Biology of Neurodegeneration, EPFL (Lausanne, Switzerland) and synthesized and purified as TFA salts by Elliott, WM Keck Facility, Yale University (New Haven, USA) as described in the literature. ${ }^{[18]}$

The preparation of $A \beta(1-42)$ monomer was performed by dissolving the peptide in $6 \mathrm{M}$ guanidine $\mathrm{HCl}$ and purifying it via size exclusion chromatography (SEC). For this purpose, the column Superdex 75 10/300 GL (GE Healthcare, Uppsala, Sweden) was used. A $\beta(1-42)$ protofibril (PF) was obtained by dissolving the peptide in $100 \%$ DMSO and diluting the solution in ultrapure $\mathrm{H}_{2} \mathrm{O}$, then purifying the obtained solution via $\mathrm{SEC}$, using the chromatographic column described above. ${ }^{[9]}$ The concentration of the samples was deter- mined by UV/Vis spectroscopy, using a molar extinction coefficient of $1490 \mathrm{M}^{-1} \mathrm{~cm}^{-1}$ at $280 \mathrm{~nm}$. Buffers and solvents for peptide preparation and SEC were purchased from SIGMA Aldrich and Fluka AG (Buchs, Switzerland). The preparation of $A \beta(1-42)$ fibril (F) was performed by incubating the monomer sample at $37^{\circ} \mathrm{C}$ for $48 \mathrm{~h}$. The procedure for $A \beta(1-42)$ monomer, $P F$ and $F$ production was carried out according to the protocol developed by Jan et al. ${ }^{[9]}$

TEM imaging was performed depositing $2 \mu \mathrm{L}$ of peptide samples on Formvar/carbon-coated TEM grids negative stained by means of uranyl acetate $2 \% .{ }^{\left[{ }^{[9]}\right.}$ The preparation of the TEM grids was carried out soon after sample preparation and then after different time lapses (from time 0 to $48 \mathrm{~h}$ for the monomer, at time 0 and $24 \mathrm{~h}$ for the protofibril, and at time 0 for the fibril) during peptide incubation at $37^{\circ} \mathrm{C}$. Both the grids and the stain were purchased from Electron Microscopy Sciences (Hatfield, USA). Micrographs were acquired on a Philips CM200 Transmission Electron Microscope.

\section{A $\beta$ (1-42) Aggregation Analysis with an Approach Based on Bloch Surface Waves}

Periodic stacks of dielectric materials (known as photonic crystals) with different refractive index constants can sustain optical surface modes ${ }^{[19]}$ with either transverse electric (TE) or transverse magnetic (TM) polarization. Such modes are called Bloch surface waves (BSWs) and have a significant amount of their overall power confined close to the truncation interface of the photonic crystal. ${ }^{[20]}$ In our experiment, the photonic structure is a multilayer made of alternating layers of silicon oxinitride and silica, as previously reported. ${ }^{[21]}$ The multilayer is deposited on a glass substrate with an Oxford Plasmalab $80+$ PECVD machine. The top layer, which is in contact with the sensed media, is silicon oxinitride.

The experimental apparatus developed in-house consists of a prism-based goniometric setup, as depicted in Figure $3 \mathrm{~A}$, wherein a proper silicon-based multilayer is used as a photonic structure sustaining BSWs. A TM-polarized laser (wavelength $632.8 \mathrm{~nm}$ ) source is first collimated, then focused (lens focal length $40 \mathrm{~mm}$, diameter $165 \mathrm{~mm}$ ) and expanded on the multilayer through the side facet of a prism. The angle of incidence of the beam on the prism can be varied in a controlled way. The glass substrate hosting the multilayer is optically contacted to the prism by means of

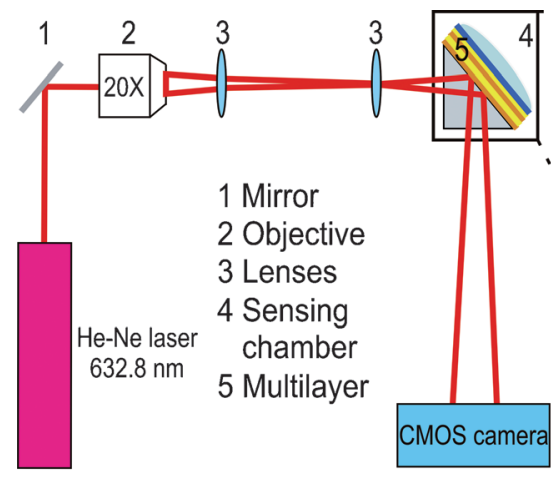

A

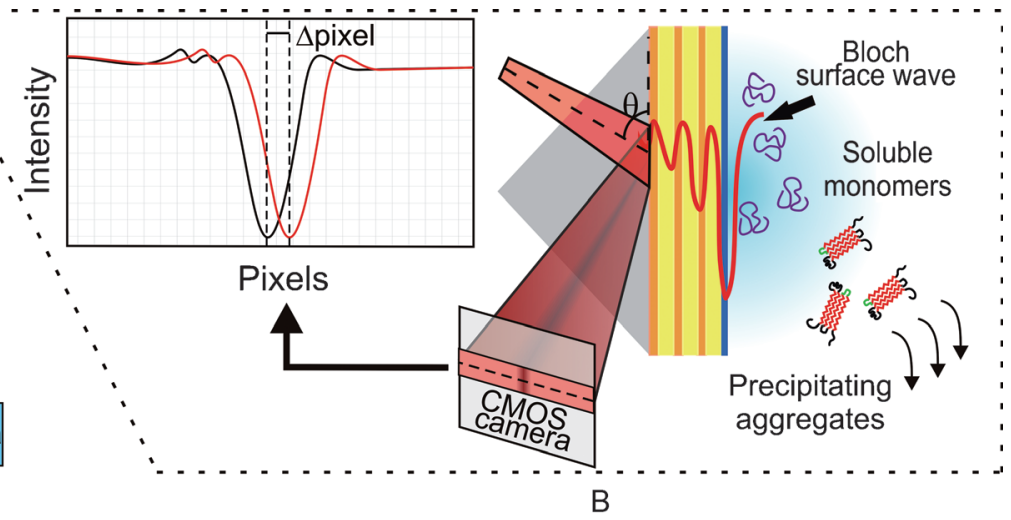

B

Figure 3. A) Schematic top-view of the optical setup. A He-Ne laser (1) is used to illuminate the photonic crystal contacted to a prism (4) and the beam is conditioned by using an objective (2) and two subsequent lenses ( 3 ) in order to obtain the divergent incident beam whose optical rays are incident on the multilayer at different angles. The light is then reflected by the multilayer (5) towards the CMOS camera. B) Lateral enlargement of the sensing chamber. The microfluidic cell is positioned vertically. The laser beam generates the BSW probing the protein solution via an evanescent tail of its associated electromagnetic field distribution. As for SPR, the coupling is observed as an intensity dip in the reflected angular distribution (represented in dark). Any variation in the refractive index of the liquid is resulting in a shift of the reflection minimum (in pixel). 
an index-matching oil, according to the attenuated total reflection configuration. As the laser impinges on the multilayer at the specific BSW resonant angle, part of the laser power is transferred to the BSW mode, resulting in an evanescent field extended in the external (aqueous) medium for a distance of about $0.5 \mu \mathrm{m}$. As a result, the corresponding reflected spot shows a decrease of light intensity (resonance dip). The reflected spot containing the BSW resonance dip is leaving the prism with a given angular divergence, and is imaged on a linear camera. The position of the dip on the linear camera is indicative of the angular position of the BSW resonance dip and hence of the refractive index as sensed by the BSW. The sensing approach is similar to the well-known SPR scheme. ${ }^{[22]}$ The truncation interface of the photonic multilayer is sealed to a fluidic cell, in which a thermocouple and a Peltier element in closed feedback loop can maintain a constant temperature.

In the present work, we exploit BSWs to locally probe the refractive index variations $(\Delta n)$ of a solution wherein the $A \beta(1-42)$ peptide is initially injected in a monomeric form and is progressively aggregating to form fibrils (Figure $3 \mathrm{~B}$ ). During this process, the $A \beta(1-42)$ peptide tends to precipitate away from the multilayered surface, since the sensing chamber is vertically positioned. As refractive index perturbations occur within the fluidic cell, the position of the BSW dip shifts. The angular position of the dip moves toward larger output angles as the refractive index increases (see Figure $3 \mathrm{~B})$.

The incubation of samples is performed at $37^{\circ} \mathrm{C}$ (uncertainty $\pm 0.1^{\circ} \mathrm{C}$ ) via the use of a Peltier thermoelectric thermostat system connected to a temperature controller Lightwave ILX (Bozeman, USA). The data acquisition of the BSW dip resonance position is started $1 \mathrm{~min}$ after the injection of the sample solution in the cell, in order to stabilize the temperature of the fluidic cell. The laser beam reflected from the multilayer is directed to the detector, a 1.3 MP Monochrome 8-bit CMOS Camera purchased from Mightex (Toronto, Canada), which allows monitoring the angular shift of the resonance conditions through the camera pixels. The response will thereforebe reported in numbers of pixels (Figure $3 \mathrm{~B}$ ).

For the results presented in this work, it is important to stress that the zero value in pixel shift corresponds to the BSW resonance position at the beginning of the measurement, and not to the signal of the reference buffer solution $(10 \mathrm{~mm}$ Tris $\mathrm{HCl})$. To guarantee identical initial experimental conditions, a new multilayer was used for each different measurement. The optical response is tested before each measurement in order to assess the BSW robustness. In order to calibrate the response of the BSW dip shift upon refractive index perturbations in the solution, ${ }^{[23]}$ control experiments were performed using solutions with different known refractive indices. For our experimental refractive index range, the result is a linear relationship between the observed shift and the refractive index of the solution as predicted by known formulas. ${ }^{\left[{ }^{[b]}\right.}$ If we estimate the sensitivity as $n^{\circ}$ pixel RIU ${ }^{-1}$ (refractive index units), the obtained experimental sensitivity is $S_{\text {pixel }}=12778$ pixels $\mathrm{RIU}^{-1}$. The experimentally observed resolution is $\Delta n=1.5 \times 10^{-4} \mathrm{RIU}$, which is sufficient for our measurements.

\section{Polyethylene Glycol Self-Assembled Monolayer Coating}

All the reagents and solvents were purchased from SIGMA Aldrich and Fluka AG (Buchs, Switzerland), and Honeywell (Seelze, Germany). Methoxy polyethylene glycol amine 750 was purchased from SIGMA Aldrich. The chemical modification of the multilayer surface was performed with a method described elsewhere. ${ }^{[2]}$ Methoxy polyethylene glycol amine 750, an $\alpha$ - and $\omega$-substituted PEG derivate, was linked to the silicon oxinitride top layer of the multilayer. The silicon oxinitride surface was treated with piranha solution (3:1
$\mathrm{H}_{2} \mathrm{SO}_{4}: \mathrm{H}_{2} \mathrm{O}_{2}$ ) that generates silanols functions $\mathrm{Si}-\mathrm{OH}$ able to react with amino-propyl-triethoxy-silanes (APTES). The free amine groups of APTES react with an N-hydroxysuccinimide (NHS) ester function of the acetal protected linker. The acetal protecting group of the linker is subsequently deprotected liberating the aldehyde. The amino end-group of the modified PEG molecule is reductively coupled to the carbonyl group of the linker. The homogeneity of the resulting PEG coating was confirmed using in-dark-field optical microscopy.

\section{Acknowledgements}

We thank Dr. Laura Chantada Santodomingo and Dr. Elsie Barakat for help with data analysis and Figure preparation and Dr. Claudio Dalvit for thoughtful discussions.

Keywords: aggregation - Alzheimer's disease $\cdot$ amyloid betapeptides $\cdot$ biosensing $\cdot$ photonic crystals

[1] J. Hardy, D. J. Selkoe, Science 2002, 297, 353-356.

[2] a) H. A. Lashuel, D. Hartley, B. M. Petre, T. Walz, P. T. Lansbury, Nature 2002, 418, 291-291; b) C. Haass, D. J. Selkoe, Nat. Rev. Mol. Cell Biol. 2007, 8, $101-112$.

[3] a) J. D. Harper, P. T. Lansbury, Annu. Rev. Biochem. 1997, 66, 385-407; b) J. W. Kelly, Nat. Struct. Biol. 2000, 7, 824-826; c) M. R. Nilsson, Methods 2004, 34, $151-160$.

[4] a) H. Li, F. Rahimi, S. Sinha, P. Maiti, G. Bitan, K. Murakami in Encyclopedia of Analytical Chemistry, Vol. S1-S3 (Ed. R. A. Meyers), John Wiley, Chichester, 2009, pp. 635-666.; b) K. A. Bruggink, M. Müller, H. B. Kuiperij, M. M. Verbeek, J. Alzheimer's Dis. 2012, 28, 735-758.

[5] a) W. M. Robertson, J. Lightwave Technol. 1999, 17, 2013; b) E. Descrovi, T. Sfez, L. Dominici, W. Nakagawa, F. Michelotti, F. Giorgis, H. P. Herzig, Opt. Express 2008, 16, 5453-5464.

[6] M. Shinn, W. M. Robertson, Sens. Actuators B 2005, 105, 360-364.

[7] a) M. Ballarini, F. Frascella, F. Michelotti, G. Digregorio, P. Rivolo, V. Paeder, V. Musi, F. Giorgis, E. Descrovi, Appl. Phys. Lett. 2011, 99, 043302-043303; b) A. Farmer, A. C. Friedli, S. M. Wright, W. M. Robertson, Sens. Actuators B 2012, 173, 79-84; c) P. Rivolo, F. Michelotti, F. Frascella, G. Digregorio, P. Mandracci, L. Dominici, F. Giorgis, E. Descrovi, Sens. Actuators B 2012, 161, 1046-1052.

[8] a) V. N. Konopsky, E. V. Alieva, Anal. Chem. 2007, 79, 4729-4735; b) F. Giorgis, E. Descrovi, C. Summonte, L. Dominici, F. Michelotti, Opt. Express 2010, 18,8087-8093; c) A. Sinibaldi, N. Danz, E. Descrovi, P. Munzert, U. Schulz, F. Sonntag, L. Dominici, F. Michelotti, Sens. Actuators B 2012, 174, 292-298.

[9] A. Jan, D. M. Hartley, H. A. Lashuel, Nat. Protoc. 2010, 5, 1186-1209.

[10] Z. Yang, J. A. Galloway, H. Yu, Langmuir 1999, 15, 8405-8411.

[11] a) K. L. Prime, G. M. Whitesides, J. Am. Chem. Soc. 1993, 115, $10714-$ 10721 ; b) K. L. Prime, G. M. Whitesides, Science 1991, 252, 1164- 1167.

[12] a) A. Jan, O. Gokce, R. Luthi-Carter, H. A. Lashuel, J. Biol. Chem. 2008, 283, 28176-28189; b) J. S. Jeong, A. Ansaloni, R. Mezzenga, H. A. Lashuel, G. Dietler, J. Mol. Biol. 2013, 425, 1765-1781.

[13] L. C. Serpell, Biochim. Biophys. Acta Mol. Basis Dis. 2000, 1502, 16-30.

[14] a) P. T. Lansbury, P. R. Costa, J. M. Griffiths, E. J. Simon, M. Auger, K. J. Halverson, D. A. Kocisko, Z. S. Hendsch, T. T. Ashburn, R. G. S. Spencer, B. Tidor, R. G. Griffin, Nat. Struct. Mol. Biol. 1995, 2, 990-998; b) D. M. Walsh, I. Klyubin, J. V. Fadeeva, W. K. Cullen, R. Anwyl, M. S. Wolfe, M. J. Rowan, D. J. Selkoe, Nature 2002, 416, 535-539.

[15] M. Ahmed, J. Davis, D. Aucoin, T. Sato, S. Ahuja, S. Aimoto, J. I. Elliott, W. E. Van Nostrand, S. O. Smith, Nat. Struct. Mol. Biol. 2010, 17, 561 567.

[16] H. Yagi, T. Ban, K. Morigaki, H. Naiki, Y. Goto, Biochemistry 2007, 46, 15009- 15017.

[17] S. A. Funke, Int. J. Alzheimer's Dis. 2011, 151645. 
[18] T. Sato, P. Kienlen-Campard, M. Ahmed, W. Liu, H. Li, J.I. Elliott, S. Aimoto, S. N. Constantinescu, J.-N. Octave, S. O. Smith, Biochemistry 2006, 45, 5503-5516.

[19] P. Yeh, A. Yariv, A. Y. Cho, Appl. Phys. Lett. 1978, 32, 104- 105.

[20] R. D. Meade, K. D. Brommer, A. M. Rappe, J. D. Joannopoulos, Phys. Rev. B 1991, 44, $10961-10964$.

[21] V. Paeder, V. Musi, L. Hvozdara, S. Herminjard, H. P. Herzig, Sens. Actuators $B$ 2011, 157, 260-264.

[22] W. L. Barnes, A. Dereux, T. W. Ebbesen, Nature 2003, 424, 824-830.
[23] E. Descrovi, F. Frascella, M. Ballarini, V. Moi, A. Lamberti, F. Michelotti, F. Giorgis, C. F. Pirri, Appl. Phys. Lett. 2012, 101, 131105-131104.

[24] L. Wildling, B. Unterauer, R. Zhu, A. Rupprecht, T. Haselgrübler, C. Rankl, A. Ebner, D. Vater, P. Pollheimer, E. E. Pohl, P. Hinterdorfer, H. J. Gruber, Bioconjugate Chem. 2011, 22, 1239-1248.

Received: July 8, 2013

Published online on September 17, 2013 